\title{
The Psychology of Flow, Mathematics Pedagogy, and Culture
}

\author{
Yvette d'Entremont ${ }^{1}$, Michelle Voillot ${ }^{2}$ \\ ${ }^{1}$ University of Alberta, Edmonton, Canada \\ ${ }^{2}$ Oceanside, California, USA
}

\begin{abstract}
The primary purpose of this paper is to illustrate how the psychological concept of flow can be applied to the teaching of mathematics through cultural artefacts. Flow has been found to increase student engagement in various educational settings. The eight characteristics of flow are outlined, followed by an overview of the use of flow within the mathematics classroom. Achieving a state of flow results in an increase in interest, motivation and therefore in students' learning. Linking mathematics to personal experiences and cultural practices have a positive effect on motivation and understanding becomes easier. Culture is a vital part of learning mathematics and cultural practices such as weaving can be motivating and lead to a better understanding of mathematics. Mathematics becomes something real and applicable to community practices and activities. The weaving activity in this paper demonstrates how linking a mathematical activity to a cultural artefact can motivate students and result in a state of flow. The experience of flow is subjective in nature. Achieving a state of flow is an individual experience and not all students will achieve a state of flow from the same activity. For this reason, educators must themselves understand the concept and the characteristics of flow if they are to lead their students to achieving flow.
\end{abstract}

\section{Introduction}

Mathematics has been perceived as a difficult subject by many students. It is often seen as a set of rules, algorithms, and theorems to memorize [4], [7]. Learning mathematics under these impressions can be burdensome, challenging, and even traumatic [8]. Many adults report having bad experiences with mathematics in school, resulting in a negative relationship with mathematics throughout the rest of their lives [8]. Attitudes towards mathematics tend to worsen throughout childhood and adolescence, hindering development, education, and engagement in mathematics-related activities [17]. Thus, student engagement in mathematics education is problematic [2].

The learning and teaching of mathematics has been influenced by many factors through the decades. One factor is cognitive psychology which is concerned with the process of learning. Another factor is the fact that today's classrooms are multicultural. It would probably be easier to teach mathematics to a group of students with similar abilities, similar learning styles, and similar cultural realities. This would also be very boring. The diversified sociocultural backgrounds of students makes teaching more interesting and more challenging. Good teaching requires a strong knowledge of the subject matter, an understanding of the psychology of learning, and the ability to lead students to the comprehension of mathematical concepts through action learning using personal cultural practices that students can relate to. Interpreting the relationship between school mathematics and culture provides a basis for student participation in their learning.

For the purpose of this paper, we concentrate on the positive psychological concept of flow. Flow, defined as a state of deep absorption in an activity that is intrinsically rewarding and enjoyable [11], has been found to increase student engagement in various educational settings [25], [5], [10], [16], [3]. Csikszentmihalyi [12] suggests that educators can help students want to learn mathematics by turning mathematics activities into flow experiences. He states that when an experience becomes intrinsically rewarding, students are motivated, engaged, and selfdirected in their acquisition of knowledge.

\section{Flow: The psychological state of optimal experience}

When musicians, athletes, and artists are fully engaged in what they are doing, and barely conscious of their surroundings, they are said to be in a state of flow. The concept of flow, a cornerstone of positive psychology, was discovered by Dr. Mihaly Csikszentmihalyi, a Hungarian psychologist best known for his work on flow, happiness, and creativity [6]. A prisoner during World War II, he wondered how individuals that experienced pain and suffering were able to find happiness and create a life worth living. By observing people working relentlessly on tasks and not bothered by boredom or fatigue, he found that happiness can be consciously cultivated 
and maintained through a state of flow [11]. While interviewing athletes, musicians and artists in order to determine how they experienced optimal performance levels, he noticed their work "flowed" out of them, leading them to their best creations and achievements. He also determined that flow requires a high level of dedication and persistence [14].

Flow is considered an 'autotelic experience' resulting from an activity that produces its own intrinsic motivation, rewards, and incentives without extrinsic benefits [16]. Numerous studies have evaluated the benefits of flow states, including increased creativity, happiness, and productivity [14]. Hou [19] determined that flow states affect student learning behavior patterns related to in-depth processes, Brom, et al. [9] found that flow increased positive affect, and dos Santos, et al. [16] stated that flow states resulted in an increase in students' learning, in-depth reflective process, and student satisfaction.

While any task has the potential for flow, certain conditions must be met prior to entering a flow state. These include a challenge-skill balance, a merging of action and awareness, a clear set of goals, unambiguous feedback, concentration on the task at hand, a sense of control, a loss of self-consciousness, and a transformation of time [6].

\section{Flow in mathematics}

A significant body of literature outlines the concept of flow yet very little research explores flow within a specific educational setting, such as learning mathematics. A number of studies have however dealt with the topic. Golnabi [18] and Chiru [10] have examined the characteristics of flow involved in the learning of mathematics. Allan [1] conducted a small research project that confirmed mathematical flow is a valid construct and concluded that "mathematical flow is a positive experience of full engagement with mathematics where joy is experienced through solving challenging problems, seeking understanding, and constructing proofs" (p.14). They found that a single mathematical flow experience can result in an increased enjoyment of mathematics and a long-term increase in engagement with mathematics. Specifically, student participants in their study indicated they experienced an intense focus on a task where nothing else mattered and a desire to keep working in order to build on new and existing knowledge to solve the next problem. Mathematical flow should provide a positive experience where mathematics is learned through activities that ensure the student is neither bored or overwhelmed by the challenge of the activity [1].

\section{Designing flow-inducing activities}

Based on the eight characteristics of flow, several researchers have put forth suggestions for inducing flow states in educational settings, including in mathematics education.

\subsection{Establishing interest}

Interest is a fundamental aspect of flow experiences as it provides the foundation for becoming engaged with a topic for its own sake [26]. Csikszentmihalyi [13] indicates that "the more difficult a mental task, the harder it is to concentrate on it. But when a person likes what he does and is motivated to do it, focusing the mind becomes effortless even when the objective difficulties are great" (p.27). Spencer [27] states that "one of the best ways to intrinsically motivate students is to connect assignments to their interests" (p.12). It has been documented that students' learning is influenced by their cultural background [31], [22], [28]. Integrating references to home and community into the subject matter can make mathematics more meaningful, more relevant, and therefore more motivating [22], [23], [15]. A mathematical task is likely to develop motivation if it sparks student interest [1]. Such referents can lead to student engagement, the first step to achieving a state of flow.

\subsection{Balance of challenge and skills}

Flow states require a balance of challenge and skills. Kiili et al. [20] state that flow experiences usually occur when a person's mind or body is stretched to its limits in a voluntary effort. Often, both skill and challenge level must be moderately high in order to experience flow [14]. A mismatch in balance and skills can result in different emotional states, such as anxiety and stress when challenge is too high, and boredom and apathy when a task is too easy [10]. Surprisingly, pressure and stress were found to trigger flow experiences [1], supporting the notion that challenge is an important aspect for flow. Although stressful, participants reported the experience as being emotionally demanding yet rewarding as they reached new levels of understanding. According to Golnabi [18], mathematical problem solving provides an appropriate context for flow to occur, since both challenges and skill level are measurable in a mathematical context. Allan [1] found that problems were best at inducing a state of flow when they had multiple solutions for variable skill levels. In other words, they are open-ended, having a low entry and a high ceiling [8]. 


\subsection{Feedback}

Flow states require consistent feedback [14]. If a student is unsure that their work is on the right path, they are easily discouraged to continue. However, if a student has access to timely feedback, they are more likely to feel motivated to finish their work. Feedback can be obtained via discussions with the instructor, group work with peers, or a clear set of assignment guidelines. In order to maximize feedback, it is important that students are able to share their mathematical thinking without fear of judgment as mistakes are to be expected and should be seen as a part of learning mathematics [8].

\subsection{Goals}

Flow states also require a clear, attainable goal [14]. Some students have difficulties portioning their work into smaller, more achievable steps. If a student attempts a task that is too large in scope, they can be easily discouraged. If a student accomplishes a series of small tasks, they are more motivated to continue their work. Allan [1] determined that mathematical flow experiences were goal driven with an aim to solve a complex problem, prove something, or understand a complex situation. Shernoff et al. [26] indicate that goals are an important precondition to flow, but only if the goals are related to the task and not imposed externally as a performance criterion.

\subsection{Control}

Flow experiences tend to come from doing math activities rather than listening to explanations [25], [1]. Waterman et al. [30] suggest that the importance of student choice is related to the necessity for control in achieving a flow state. According to Golnabi [18], being in control is central to the experience of mathematical flow. As such, interventions and activities should be designed to maximize student choice and control. Chiru [10] was able to determine that a balance between demands and control is as important as the balance between challenge and skills, suggesting that increases in challenge should also be accompanied with increase in decision latitude for students.

Allan [1] ascertained that questions which best encourage mathematical flow states were open-ended, involved student choice and required the use of student creativity. Flow was highest when activities focused on conceptual understanding and proofs with complex and challenging open-ended questions, as opposed to questions with predetermined procedural answers. These open-ended tasks allowed students to make choices and gain more control, both important for both flow and intrinsic motivation.

Flow experiences tend to come from doing math activities rather than listening to explanations [25],
[1]. Waterman et al. [30] suggest that the importance of student choice is related to the necessity for control in achieving a flow state. According to Golnabi [18], being in control is central to the experience of mathematical flow. As such, interventions and activities should be designed to maximize student choice and control. Chiru [10] was able to determine that a balance between demands and control is as important as the balance between challenge and skills, suggesting that increases in challenge should also be accompanied with increase in decision latitude for students.

Allan [1] ascertained that questions which best encourage mathematical flow states were open-ended, involved student choice and required the use of student creativity. Flow was highest when activities focused on conceptual understanding and proofs with complex and challenging open-ended questions, as opposed to questions with predetermined procedural answers. These open-ended tasks allowed students to make choices and gain more control, both important for both flow and intrinsic motivation.

\subsection{Environment}

Classroom environment can affect the prevalence of mathematical flow. A friendly and supportive classroom environment where mistakes are seen as learning opportunities results in a greater likelihood of experiencing flow [1]. Additionally, such an environment can trigger group mathematical experiences. For instance, Allan [1] and Armstrong [3] found that group flow facilitated learning and progress that could not have been achieved individually. Group flow was strongest when the environment contained whiteboards for the students to use, allowing them to easily share their ideas with their peers, to have the freedom of movement to collaborate, and to quickly erase when necessary. However, Armstrong [3] cautions that the environment should support sufficiently decentralized groups in order to experience group flow.

\section{Weaving: A flow-inducing activity}

Motivation is an important factor when learning mathematics and is directly related to the quality of the experience. Students who are not motivated will not be interested and therefore the experience will be negative or neutral at best. A large part of flowinducing activities involve social interactions between students and within the activity itself. Connections between the culture and community of the students and the subject matter motivate and encourage learning making mathematics meaningful. Weaving is an activity that is familiar to many students as it is an important practice in all cultures. 
Weaving is a broad term used to encompass a variety of skills such as basket weaving, art hangings such as tapestries, rug making, and the production of textiles. It is a skill that is valuable in many cultures around the world and examples of this can be seen in the artefacts and practices of various ethnicities. Weaving in the production of textiles is a process by which yarns of various colors are interlaced in such a way as to create a design or pattern that repeats. Motifs in woven cloth are an important part of every culture and vary within communities. These fabrics are used for a variety of purposes: practical, symbolic, religious or ceremonial. Textiles, their creation and their uses, play an important part in studying history, culture and mathematics. Tartans are an example of weaving motifs that have cultural and historical significance. How many Canadian students know that Canada has a national tartan, the Maple Leaf tartan and can recognize it (see Figure 1)? Studying the historical aspect of the Maple Leaf will lead to not only a discussion regarding its historical significance, the meaning of the colors, but also to an analysis of the design.

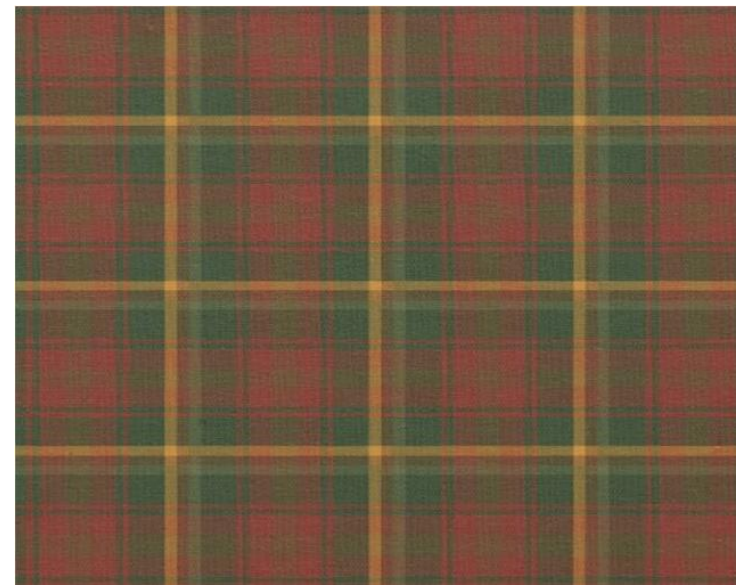

Figure 1. Maple Leaf tartan

Other tartans or textile motifs can then be studied for their historical and cultural significance. Students should be then able to determine the significance of the colors involved in the production of this tartan. By studying their own community, they can determine which community groups and organizations have tartan as part of their formal dress and what is the significance of the pattern and colors. Community resources are an asset to learning and should not be ignored [15].

Math is perhaps not the first thing that comes to mind when thinking about weaving but weaving patterns and their connection to mathematics have been studied by various researchers such as Zaslavsky [32] and Washburn [29] who have studied patterns in African cultures. The following activity is based on the process of weaving to create a repeating pattern as would be used in textile production. Not only is this activity culturally significant, it is also a mathematical activity. Studying the pattern of a woven artifact, such as the Maple Leaf tartan, or the 'ceinture fléchée' (French sash or Métis sash) (Figure 2) can lead to a discussion about various mathematical concepts, as well as its historical and cultural significance.

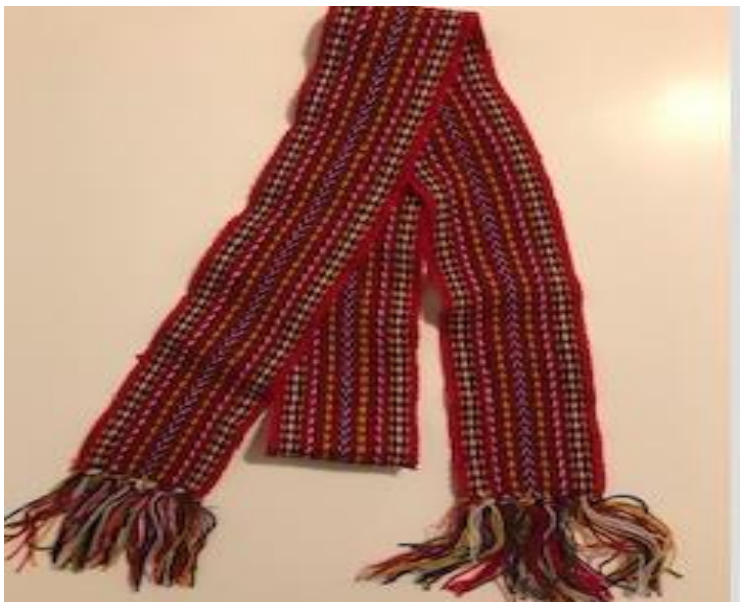

Figure 2. French Sash Métis Sash

This is also an opportunity to link school, and community. Students who have a sash at home may wish to bring it to school and talk about it's significance, how it was made, and by whom. Communication and discourse are important components of mathematics learning. Each sash can be displayed to study the patterns.

Prahmana and D'Ambrosio [21] have indicated that mathematics is part of our daily life and that culture can be used as a basis to learn mathematics. Their study explored the mathematical concept of geometry transformation in the Yogyakarta batik pattern. An analysis of various weaving patterns shows that the concept of transformational geometry has been applied.

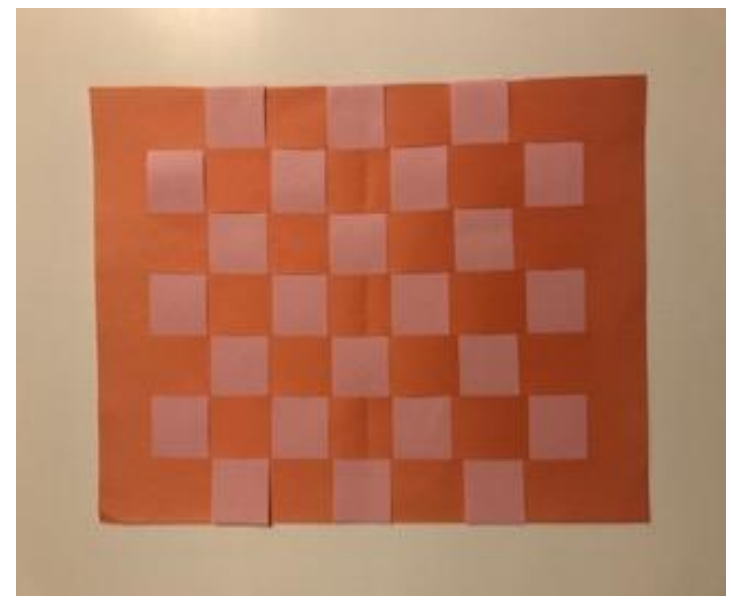

Figure 3. Simple weaving pattern 
An analysis of tartans and their significance demonstrates the use of mathematical concepts such as counting, measuring, symmetry, tessellations, problem solving, increasing and decreasing patterns, repeating patterns, and geometrical transformations.

Having understood the process of weaving, students could create their own pattern. Figures 3 and 4 are representative of the over, under, over, under weaving technique. Using colored paper, elementary school students can practice the over, under, over, under technique (see Figure 3 ) to achieve a simple design.

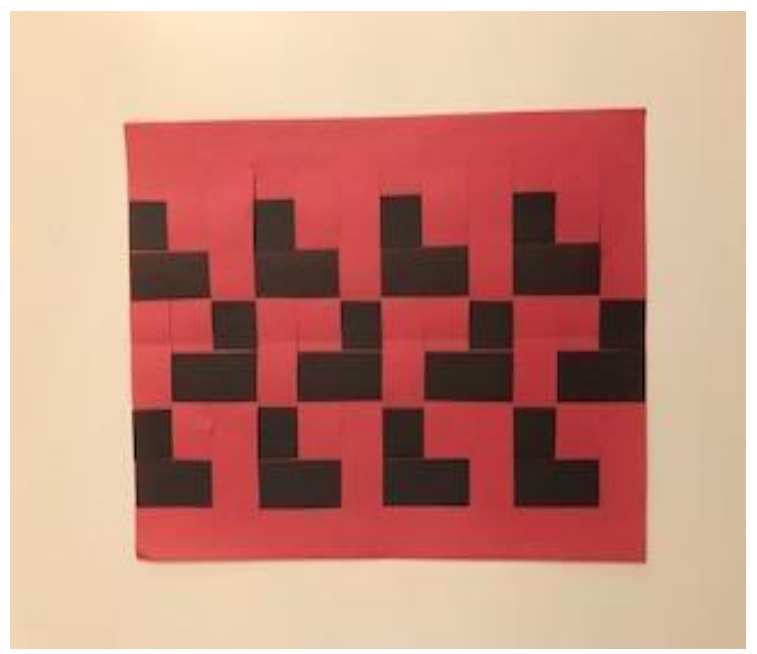

Figure 4. Weaving pattern using transformational geometry

Properties of transformational geometry have been applied in Figure 4. This pattern used the concept of geometric transformation in the form of reflection and translation. Before weaving their pattern, students can draw their design on grid paper.

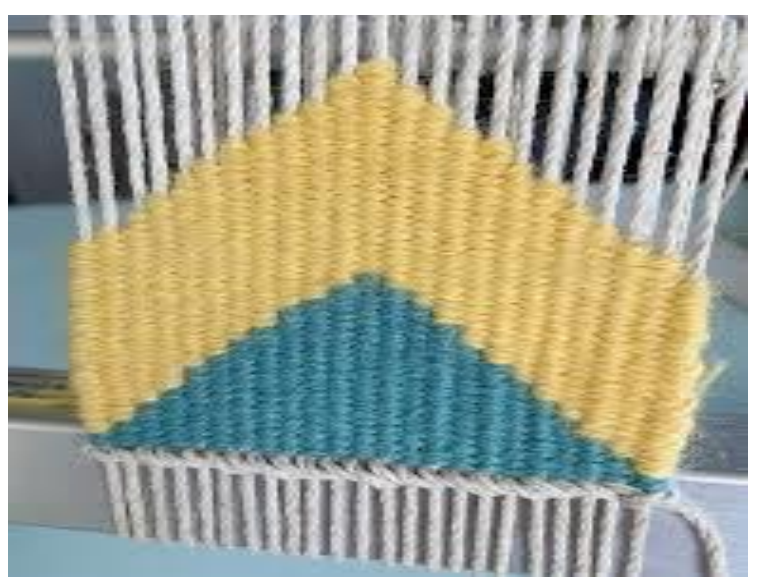

Figure 5. Increasing and decreasing pattern

Interested geometric designs can be created and integrated into discussions about the mathematics involved. An example of a design using an increasing and decreasing pattern is shown in Figure 5. All student created designs can be interpreted, analyzed, and discussed. Being able to communicate the process applied in the creation of the design is an important element to mathematics learning.

This type of activity has the potential to induce flow for several reasons. It addresses student interest by discussing aspects of their cultural and community weaving practices. By creating their own design and determining the complexity, students themselves determine the balance between their skill level and the challenge while also maintaining creative control over their own project. The goal of such an assignment is clear, and it allows for consistent feedback as students will recognize whether or not their weaving fits into their desired patterns. Students can share their project with their peers and teachers as an additional source of feedback. The level of mathematics employed in the creation of the design depends on the complexity of the design. This creates a balance between knowledge and skill level, one of the characteristics of flow. Upon reaching one level of understanding, students can increase their skill level by creating a different pattern requiring the application of other mathematical concepts. Good material acts as a stepping stone to learning mathematics. Such an activity should not simply be a fun activity but one based on mathematical reasoning.

\section{Discussion}

Mathematics cannot be considered a culture free discipline. It is an integral part of cultural activities, and practices [15]. All cultures are rich in practices and artefacts that demonstrate a strong knowledge of mathematical concepts. Educators incorporating cultural artefacts in their teaching practices can explore mathematics from a different perspective. Using relevant cultural artefacts which students can analyze, interpret, and recreate their own way, provides opportunities for being creative and using their mathematical knowledge. The weaving activity demonstrated using geometric transformations can be interlaced with the characteristics of flow. An artefact that students have seen, and of which most would have at home, will establish interest. Interest will first be generated by the fact that this object, the sash or the tartan is familiar and real. This provides a platform for students to discuss the creation and significance of a woven artefact. Creating a design of their own allows the student to choose the level of difficulty. The balance between challenge and skills is delicate. Student engagement is a personal thing. By allowing students to create their own design, they decide the challenge level that best fits their skill level. The feedback is immediate. The student realizes whether the pattern is as he/she planned. The goal of the task is controlled by the student. Educators must also realize that not all students will show enough interest and motivation in the same activity to achieve a flow 
state. Being in control is essential to flow [18], but does mean simply doing something fun and easy. There must be a balance between challenge and skill. This balance must be challenging enough to achieve higher mathematical learning. Not all students are able to self-evaluate and may need teacher intervention [10]. It has been determined [1] that activities that involved student choice and required the use of student creativity were activities that would best encourage flow by merging action and awareness.

\section{Conclusion}

Mathematics is more than a set of predetermined procedures resulting in right or wrong answers. It is a multidimensional subject that requires reasoning, creativity, connection making, and interpretation [8]. With flow theory, students can experience the satisfaction of learning something new. Flow experiences can contribute to a growth mindset by encouraging students to see effort as their path to mastery and to perceive mistakes as learning opportunities [1], [8]. Csikszentmihalyi [14] states "science and math, for instance, have the initial disadvantage of presenting too many challenges to students, who start out being anxious and often remain in that state without ever enjoying the learning process" (p.185). The goals, the rules, and the constant feedback provided in mathematics class are criteria that lead to a flow experience. Teaching strategies that connect with the real lives of students and their community, promote understanding leading to academic achievement. When students see that mathematics is close to the culture around them, mathematics becomes meaningful and can be applied to their lived experiences [21]. Not only does integrating a cultural artefact pertinent to students' community provide for mathematics learning, it creates an important link with the community. This link makes mathematics interesting, motivating, and real; all conditions essential to achieving a state of flow. Implementing the use of artefacts into mathematics teaching is not easy and should be considered carefully. Teachers must know how and why they are using artefacts to teach math. People do not experience the same situation in the same way, or in their desire to do what they are doing. Therefore, educators must also realize that not all students experience flow in the same manner, nor does the same activity lead to a flow experience for all students. Achieving a state of flow is an individual experience. Educators must themselves understand the concept of flow if they are to lead their students to achieving flow.

\section{References}

[1] Allan, P. (2015). Inducing mathematical flow. [Unpublished master's thesis]. Auckland University, New-Zealand.

[2] Anthony, G., and Walshaw, M. (2007). Effective pedagogy in mathematics/pāngarau: Best evidence synthesis iteration (BES). Wellington, New Zealand: Ministry of Education.

[3] Armstrong, A. C. (2008). The fragility of group flow: The experiences of two small groups in a middle school mathematics classroom. The Journal of Mathematical Behavior, 27(2), 101-115.

[4] Barkley, C., Barta, J. and Eglash, R. (2014). Math is a verb: Activities and lessons from cultures around the world. Reston, Virginia: National Council of Mathematics.

[5] Basawapatna, A. R., Repenning, A., Koh, K. H., and Nickerson, H. (2013). The zones of proximal flow: Guiding students through a space of computational thinking skills and challenges. Proceedings of the Ninth Annual International ACM Conference on International Computing Education Research.

[6] Beard, K. S. (2014). Theoretically speaking: An interview with Mihaly Csikszentmihalyi on flow theory development and its usefulness in addressing contemporary challenges in education. Educational Psychology Review, 27(2), 353-364.

[7] Boaler, J. (2008). What's math got to do with it? How teachers and parents can transform mathematics learning and inspire success. New York: Viking.

[8] Boaler, J. (2016). Mathematical mindsets: Unleashing students' potential through creative math, inspiring messages and innovative teaching. San Francisco, CA: Jossey-Bass.

[9] Brom, C., Buchtova, M., Sisler, V., Dechterenko, F., Palme, R. and Glenk, L. M. (2014). Flow, social interaction anxiety and salivary cortisol responses in serious games: a quasi-experimental study. Computers and Education, 79, 69-100.

[10] Chiru, I, M. (2017). Occasioning flow in the mathematics classroom: Optimal experiences in common places. [Unpublished master's thesis]. Simon Fraser University, British Columbia, Canada.

[11] Csikszentmihalyi, M. (1990). Flow: The psychology of optimal experience. New York: Harper Perennial Modern Classics. 
[12] Csikszentmihalyi, M. (1991). Thoughts About Education. In D. Dickinson (Ed.), Creating the future: Perspectives on educational change. Aylesbury, U.K.: Accelerated Learning Systems.

[13] Csikszentmihalyi, M. (1997). Finding flow: The psychology of engagement with everyday life. New York, NY: Basic Books.

[14] Csikszentmihalyi, M. (2014). Intrinsic motivation and effective teaching, In Applications of flow in human development and education: The collected works of Mihaly Csikszentmihalyi. Dordrecht, Netherlands: Springer, 2014.

[15] d'Entremont, Y. (2015). Linking mathematics, culture and community. Procedia-Social and Behavioral Sciences, 174, 2818-2824.

[16] dos Santos W. O., Bittencourt, I. I., Dermeval, D., Isotani, S., Marques, L. B. and Silveira, I. F. (2018). Flow Theory to Promote Learning in Educational Systems: Is it Really Relevant? Brazilian Journal of Computers in Education, 26(2), 29-59.

[17] Dowker, A., Sarkar, A., and Looi, C. Y. (2016). Mathematics Anxiety: What Have We Learned in 60 Years? Frontiers in Psychology, 7, 1-16.

[18] Golbabi, L. (2017). Mathematics self-efficacy and flow in developmental mathematics students. [Unpublished doctoral dissertation]. Columbia University.

[19] Hou, H.T. (2015). Integrating cluster and sequential analysis to explore learners' flow and behavioral patterns in a simulation game with situated-learning context for science courses: a videobased process exploration. Computers in Human Behavior, 48, 424-435.

[20] Kiili, K., de Freitas, S., Arnab, S., and Lainema, T. (2012). The Design Principles for Flow Experience in Educational Games. Procedia Computer Science, $15,78-91$.

[21] Prahmana, R.C.I. and D'Ambrosio, U. (2020). Learning geometry and values from patterns: Ethnomathematics on the batik patterns of Yogyakarta, Indonesia. Journal of Mathematics Education, 11(3), 439-456.

[22] Presmeg, N. (2007). The role of culture in teaching and learning mathematics. In F. Lester, Jr.
(Ed.). Second Handbook of research on mathematics teaching and learning. (pp.435-458). Charlotte, NC: Information Age Publishing.

[23] Rosa, M., and Orey, D. C. (2009). Challenges faced by multicultural and multilingual schools in the United States: The case of mathematics. La Salle Revista de Educacao Ciencia e Cultura, 14(1), 29-44.

[24] Rosa, M. and Orey, D.C. (2010). Culturally relevant pedagogy: An ethnomathematical approach. Horizontes, 28(1), 19-1.

[25] Shernoff, D., Csikszentmihalyi, M., Shneider, B., and Shernoff, E. (2003). Student engagement in high school classrooms from the perspective of flow theory. School Psychology Quarterly, 18(2), 158.

[26] Shernoff D.J., Csikszentmihalyi M., Schneider B., Shernoff E.S. (2014) Student Engagement in High School Classrooms from the Perspective of Flow Theory. School Psychology Quarterly, 18(2), 158176.

[27] Spencer, J. (2017). Making learning flow. Bloomington, IN: Solution Tree Press.

[28] Stylianides, A. and Stylianides, G. (2007). Learning mathematics with understanding: A critical consideration of the learning principles in the Principles and Standards for School Mathematics. The Mathematics Enthusiast, 4(1), 103-114.

[29] Washburn, D. (1991). Symmetries of culture: Theory and practice of plane pattern analysis. University of Washington Press.

[30] Waterman, A., Schwartz, S., Goldbacher, E., Green, H., Miller, C. and Philip. S. (2003). Predicting the subjective experience of intrinsic motivation: The roles of self-determination, the balance of challenges and skills, and self-realization values. Personality and Social Psychology Bulletin, 29, 1447-1458.

[31] Zaslavsky, C. (1996). The multicultural math classroom. Bringing in the world. Portsmouth, $\mathrm{NH}$ : Heineman.

[32] Zaslavsky, C. (1973). Africa counts: Number and pattern in African Culture. Brooklyn, NY: Lawrence Hill Books. 